\title{
Treatment decision-making for sporadic small vestibular schwannoma in a pediatric patient: A case report and literature review
}

\author{
JUN WANG ${ }^{1}$, YAJING XU ${ }^{1},{\text { TING } \text { LEI }^{2} \text { and LIANG ZENG }}^{2}$ \\ ${ }^{1}$ Department of Neurosurgery, Renmin Hospital, Wuhan University, Wuhan, Hubei 430030; \\ ${ }^{2}$ Department of Neurosurgery, Tongji Hospital, Huazhong University of Science and Technology, \\ Wuhan, Hubei 430060, P.R. China
}

Received May 3, 2014; Accepted January 16, 2015

DOI: $10.3892 / 01.2015 .3058$

\begin{abstract}
The current study reports the case of a 15-year-old male who presented with hearing loss due to a small left-sided vestibular schwannoma (VS) not associated with neurofibromatosis type 2 (NF2), which had been apparent for six months. Magnetic resonance imaging with gadolinium diethylenetriamine penta-acetic acid revealed a mass, $10 \mathrm{~mm}$ in diameter, located in the left inner auditory canal. The patient had no family history of NF2 and gene mutation analysis showed no signs of the condition. Small sporadic or non-NF2 VS is extremely rare and the treatment decision-making process is complicated in children when considering the implications for the impairment of childhood development and lifelong disability. Following careful consideration, the patient in the present study underwent treatment with stereotactic radiosurgery. The five-year post-operative follow-up examination showed tumor stability without additional neurological deficits and at the time of writing the patient was alive and well.
\end{abstract}

\section{Introduction}

Vestibular schwannomas (VSs) are histologically benign tumors that originate from the vestibular division of the eighth cranial nerve. The tumors may occur sporadically, most commonly in the fifth decade of life and later, or as part of the clinical complex in neurofibromatosis type 2 (NF2), which occurs bilaterally and in young individuals. Sporadic or non-NF2 VSs are extremely rare in pediatric patients (1).

Correspondence to: Dr Liang Zeng, Department of Neurosurgery, Tongji Hospital, Huazhong University of Science and Technology, 1,095 Jie Fang Road, Wuhan, Hubei 430060, P.R. China

E-mail: zengliang209@gmail.com

Key words: conservative management, vestibular schwannoma, acoustic neuroma, microsurgery, radiosurgery
Currently, there are a variety of treatment options for the management of VSs, including observation with serial imaging, radiosurgery and microsurgery (2). The range of tumor volumes and growth patterns make the management of these lesions an issue that is much debated. Due to the clinical heterogeneity among patients with VSs, no standard patient management has been reported (3). Pediatric patients with small VSs can be challenging to neurosurgeons.

In the present study we discuss the presentation, treatment course and follow-up outcome for a pediatric patient with sporadic unilateral VSs and reviewed other related literature. This study aims to explore the current treatment paradigm for sporadic Vss in the pediatric population. Written informed consent was obtained from the patient's family for publication of this case study and the accompanying images.

\section{Case report}

In March 2009, a 15-year-old male, previously in good health, presented to Tongji Hospital, Huazhong University of Science and Technology (Wuhan, China) with complaints of left-sided progressive hearing loss that had been apparent for six months. Upon examination, left-sided sensorineural hearing loss of $60 \mathrm{~dB}$ was found, with no other neurological impairments. Cerebral computed tomography revealed an enlarged inner auditory canal on the left side (Fig. 1A). Magnetic resonance imaging (MRI) with gadolinium diethylenetriamine penta-acetic acid demonstrated a mass that was entirely located in the inner auditory canal, measuring $10 \mathrm{~mm}$ at its largest diameter (Fig. 1B). Homogeneous enhancement was documented on contrast MRI. The patient had no family history of NF2 and gene mutation analysis showed no signs of the condition. Following a discussion on the risks and benefits of microsurgery versus $\gamma$-knife surgery, and subsequent to conservative observation with a series of images, the patient chose radiosurgery treatment, which was administered once at a dose of 11.7 Gy. Following radiosurgery, the patient was discharged with left-sided hearing loss. No other neurological complaints or findings were observable on a physical examination. At 36 months post-treatment, the patient exhibited no identifiable tumor enlargement. 

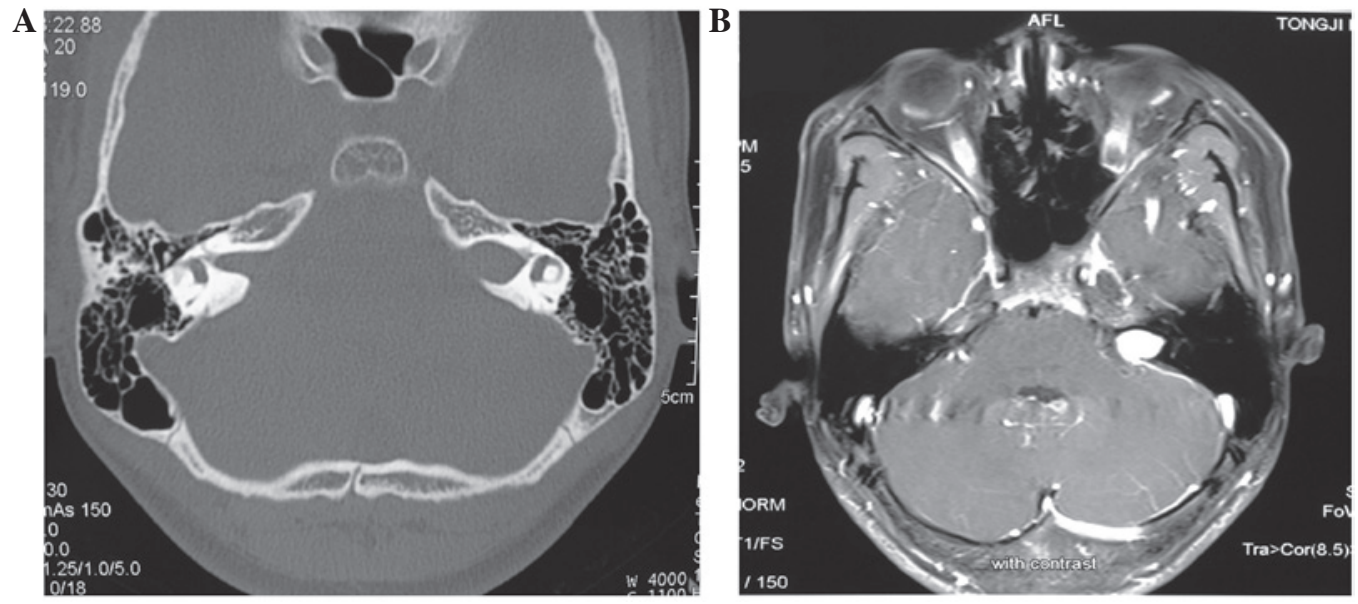

Figure 1. Upon admission, (A) axial computed tomography demonstrated an enlarged inner auditory canal on the left side, and (B) axial T1-weighted magnetic resonance imaging with gadolinium diethylenetriamine penta-acetic acid revealed a vestibular schwannoma that was entirely located in the left inner auditory canal and measured $10 \mathrm{~mm}$ at its largest diameter.

\section{Discussion}

VSs represent $0.8 \%$ of all tumors in pediatric patients ( $<16$ years of age). They are more common in young males than young females, although VSs are more common in females overall (4). The present study reports a case of VS in a male patient. An extensive literature review reveals only one reported case of small $(\leq 10-\mathrm{mm})$ non-NF2 VS in the pediatric population as part of a case series (5). Furthermore, Walcott et al (1) reported seven cases of larger VSs in the pediatric population, Holman et al (4) reported 20 cases of small pediatric VSs with NF2 and 13 cases of larger sporadic VSs in the pediatric population and Hueng et al (6) reported three adult cases of sporadic VSs. The present study is the first case report with a clinical description and radiological images of this entity. Unlike in adults, children may easily neglect the early symptoms of VS, such as unilateral hearing loss or tinnitus; instead the tumors are often identified by the more overt signs caused by the space-occupying nature of the tumor. This delay in diagnosis, usually for longer than months or years, has often resulted in tumors that are large in size (5).

Within the literature, the treatment strategies for small VSs are varied, making it difficult for a neurosurgeon to decide the best course of action $(4,7,8)$. In a study from the US, the strongest predictors of treatment modality were patient age and tumor size (3). The treatment decision rests on the availability of information with regard to the characteristics of the patient and the tumor. The decision is often hard due to inconsistencies in the data on the natural history of VS, the mechanism of hearing loss, and the hearing and facial function preservation rates associated with treatment (9). This variability is a major obstacle to practicing evidence-based medicine. The dilemma in treatment decision-making is complicated in children due to implications for the impairment of childhood development and lifelong disability. The first concern is preservation of facial nerve function, which is paramount, particularly in children, as it is often regarded as the most important index for quality of life post-operatively (5).
It has been suggested that certain factors, including the preference of the patient toward risk-aversion, the relative weight of discomfort at the present time versus the possible loss of function in the future, and the degree of comfort with regard to an uncertain long-term prognosis following radiation or during observation, will heavily effect the treatment decision (10). For the present patient, a summary of information on all three management options was provided. While radiosurgery was recommended to this pediatric patient with a small tumor, observation and surgery were also discussed, and the pros and cons of these treatment options were conveyed. This dialogue can avoid later dispute concerning the treatment of other individuals using means that the patient had not been told about, which can easily lead to distrust and even discrepancy.

All three treatment options may have been of optimal use for this case in the present medical environment. More objective data on treatment comparisons in VS management in the future will provide further evidence to aid patients and neurosurgeon in the decision-making process with a rational basis. Outcome measures should include quality of life, using a scaling system specifically designed for this entity. The treatment strategies for small VSs in pediatric patients should be tailored to the clinical, personal and social requirements of the patient. However, a rigid treatment protocol is not practical at present, and the treatment decision-making for this type of lesion is always an art form in itself.

To conclude, in the present case the treatment method without randomized controlled trial evidence was chosen on an individual basis, taking into consideration the patient's preferences, clinician experience and the availability of radiosurgical equipment. $\gamma$-knife radiosurgery was confirmed to provide long-term tumor control for sporadic small sized vestibular schwannoma in pediatric patients. The risk for loss of hearing is high, whereas the risk for other cranial nerve complications is low. Therefore, radiosurgical treatment should be considered for the management of sporadic small VS in pediatric patients if imaging indicates a small tumor size. Future studies using large patient cohorts are required to establish a standard treatment procedure for this condition. 


\section{References}

1. Walcott BP, Sivarajan G, Bashinskaya B, Anderson DE, Leonetti JP and Origitano TC: Sporadic unilateral vestibular schwannoma in the pediatric population. Clinical article. J Neurosurg Pediatr 4: 125-129, 2009.

2. Araiza Navarrete JA, Aquirre Amador LA, Escamilla Ocañas CE, Martínez Menchaca HR, Treviño Alanís MG and Rivera Silva G: Vestibular schwannoma. Gac Med Mex 148: 192-193, 2012 (In Spanish).

3. Babu R, Sharma R, Bagley JH, Hatef J, Friedman AH and Adamson C: Vestibular schwannomas in the modern era: epidemiology, treatment trends, and disparities in management. J Neurosurg 119: 121-130, 2013.

4. Holman MA, Schmitt WR, Carlson ML, Driscoll CL, Beatty CW and Link MJ: Pediatric cerebellopontine angle and internal auditory canal tumors: clinical article. J Neurosurg Pediatr 12: 317-324, 2013

5. Mazzoni A,Dubey SP,Poletti AM and Colombo G: Sporadic acoustic neuroma in pediatric patients. Int J Pediatr Otorhinolaryngol 71: 1569-1572, 2007.
6. Hueng DY and Sytwu HK: Vestibular schwannoma growth and hearing loss. J Neurosurg 120: 786, 2014.

7. Hasegawa T, Kida Y, Kato T, Iizuka H, Kuramitsu S and Yamamoto T: Long-term safety and efficacy of stereotactic radiosurgery for vestibular schwannomas: evaluation of 440 patients more than 10 years after treatment with Gamma Knife surgery. J Neurosurg 118: 557-565, 2013.

8. Hayashi M, Chernov MF, Lipski SM, Tamura N, Yomo S, Horiba A, Tsuzuki S, Izawa M, Okada Y, Muragaki Y, et al: Do we really still need an open surgery for treatment of patients with vestibular schwannomas? Acta Neurochir Suppl 116: 25-36, 2013.

9. Robinett ZN, Walz PC, Miles-Markley B, Moberly AC and Welling DB: Comparison of long-term quality-of-life outcomes in vestibular schwannoma patients. Otolaryngol Head Neck Surg 150: 1024-1032, 2014.

10. Rutherford SA and King AT: Vestibular schwannoma management: What is the 'best' option? Br J Neurosurg 19: 309-316, 2005. 